\title{
Confined Three-Dimensional Plasmon Modes inside a Ring-Shaped Nanocavity on a Silver Film Imaged by Cathodoluminescence Microscopy
}

\author{
X. L. Zhu, Y. Ma, J. S. Zhang, ${ }^{*}$ J. Xu, X. F. Wu, Y. Zhang, X. B. Han, Q. Fu, Z. M. Liao, L. Chen, and D. P. Yu \\ State Key Laboratory for Mesoscopic Physics, Department of Physics, Peking University, \\ Beijing 100871, People's Republic of China
}

(Received 15 October 2009; published 14 September 2010)

\begin{abstract}
The confined modes of surface plasmon polaritons in boxing ring-shaped nanocavities have been investigated and imaged by using cathodoluminescence spectroscopy. The mode of the out-of-plane field components of surface plasmon polaritons dominates the experimental mode patterns, indicating that the electron beam locally excites the out-of-plane field component of surface plasmon polaritons. Quality factors can be directly acquired from the spectra induced by the ultrasmooth surface of the cavity and the high reflectivity of the silver (Ag) reflectors. Because of its three-dimensional confined characteristics and the omnidirectional reflectors, the nanocavity exhibits a small modal volume, small total volume, rich resonant modes, and flexibility in mode control.
\end{abstract}

Miniaturization is a basic requirement for future applications of nanophotonic devices and integrated optical circuits. One of the essential building blocks in the nanophotonic devices is a nanoresonator with tunable optical dispersion. Although a small mode volume can be obtained by using photonic crystals [1], the total volume of the microcavity has microscale dimensionality due to its periodic structure $(\sim \lambda)$. Surface plasmon polaritons (SPPs), which are coupled excitations of electrons bound to a metal-dielectric interface [2], show great potential for application in future nanoscale photonic systems [3]. By using the nature of field confinement of SPPs, because plasmonic cavities can have a much smaller volume and fascinating properties, several types of plasmonic resonators have been proposed [4-12].

In this Letter, we investigate the plasmonic modes of a boxing ring-shaped nanocavity on Ag film. Spatially and spectrally resolved cathodoluminescence (CL) imaging spectroscopy [13-15] is then used to measure the spectra and the plasmonic modes of the nanocavity. The difference of the reflection phase shift between the in-plane and outof-plane electronic field components of the SPPs results in different mode patterns. The metal reflectors lead to a three-dimensional confinement of the SPPs, and their height presents another degree of freedom to control the resonant mode.

The nanocavity has four $\mathrm{Ag}$ walls, which is schematically shown in Fig. 1(a). Electron beam lithography and a developed template stripping method $[16,17]$ were used to fabricate precisely the fine nanostructure, which will be discussed in detail in another paper. In the experiment settings, the height of the $\mathrm{Ag}$ walls was $300 \mathrm{~nm}$, and their thickness was $\sim 500 \mathrm{~nm}$, which is larger than the skin depth of the Ag film. The side length of the nanocavity, $L$, was changed to modulate the resonant wavelengths. Figures 1(b) and 1(c) show the top-view and tilted scanning electron microscope (SEM) images of a representative nanocavity, respectively.

The resonant wavelength was measured and the spatial patterns of the resonant modes of the nanocavities were imaged by using a CL microscope installed in a SEM detection system. This technique to investigate plasmonics has been used in previous studies [13-15]. A $30 \mathrm{keV}$ electron beam impinged on samples. The collected emission spectra include the transition radiation emission $[18,19]$ and light decoupled from the SPPs. For accuracy, the CL spectroscopic responses were corrected by subtracting the background emission spectra of unstructured $\mathrm{Ag}$ from the structured Ag sampling area. For spectrally resolved imaging, the electron beam homogeneously scans over a chosen area in a scanning operation mode. Emitted light was coupled through the grating monochromator at selected wavelengths with a spectral passband $\sim 30 \mathrm{~nm}$. In the CL monochromatic image, the intensity of each pixel directly reflects the detected light intensity of the spot when it was excited by the electron beam [15]. Any drift in the scanning process of the CL images is not corrected.

Figure 2(a) depicts the collected CL spectra of 12 different nanocavities with the cavity length increasing from 320 to $865 \mathrm{~nm}$. The electron beam was positioned along the
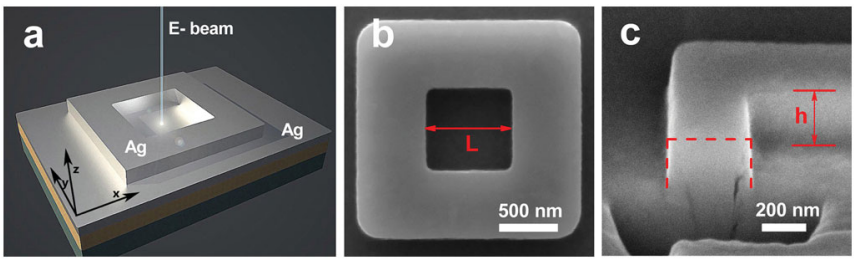

FIG. 1 (color). (a) Schematic of the nanocavity and electron beam irradiation. (b) Top-view SEM image of a cavity with a cavity length of $720 \mathrm{~nm}$. (c) $52^{\circ}$-tilted SEM image of focused ion beam milled, cross-sectional profile of a cavity. 

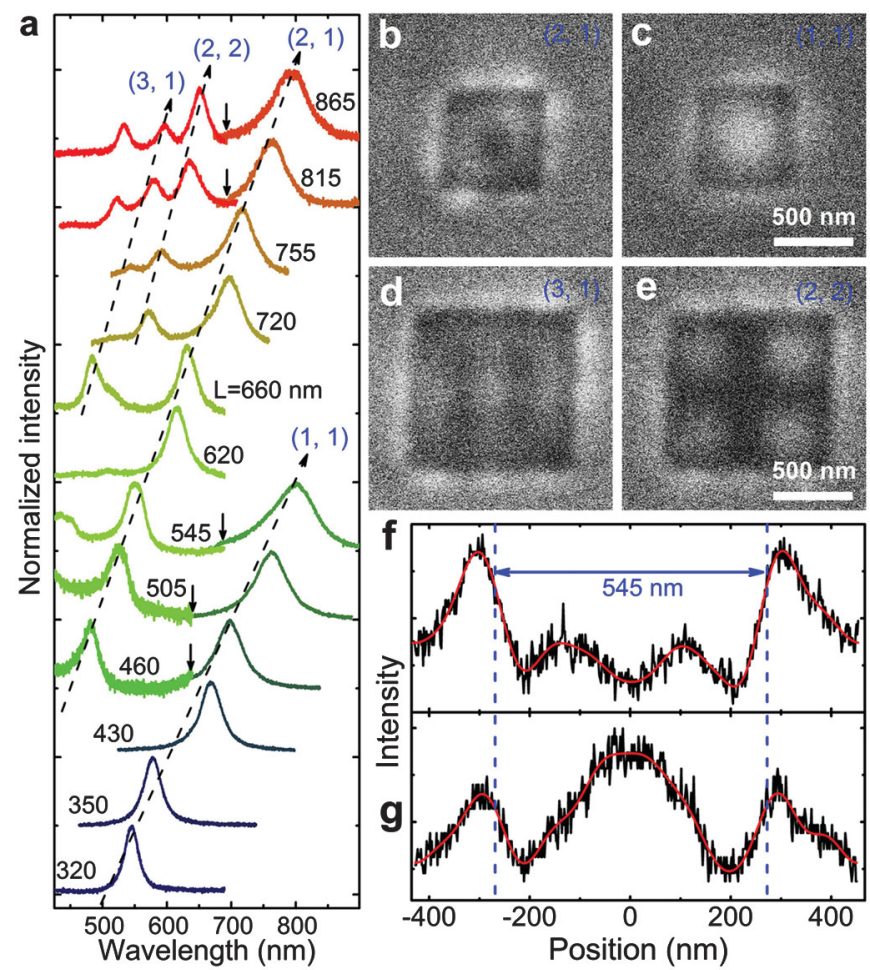

FIG. 2 (color). (a) CL spectra of 12 nanocavities with cavity length increasing from 320 (bottom) to $865 \mathrm{~nm}$ (top). The black arrows indicate the breaks of two measurements. (b),(c) Typical monochromatic CL images for plasmonic modes $(2,1)$ and $(1,1)$, respectively. (d),(e) Typical monochromatic CL images for plasmonic modes $(3,1)$ and $(2,2)$, respectively. (f), (g) Line profiles of modes $(2,1)$ and $(1,1)$ along the center of the cavity from the CL images (b) and (c), respectively.

diagonal line of the nanocavities. At each position, a corresponding spectrum was collected with an exposure time of $16 \mathrm{~s}$. The black arrows in Fig. 2(a) indicate the breaks of two measurements. Cavities with $L \leq 430 \mathrm{~nm}$ have only one peak in the detection wavelength range, which is the resonant wavelength of the lowest-order mode. Higher-order modes appear with every increase in the cavity length. Spectrally resolved CL images of the nanocavity with $L=545 \mathrm{~nm}$ at two resonant wavelengths of 550 and $800 \mathrm{~nm}$ are shown in Figs. 2(b) and 2(c), respectively. Figures 2(d) and 2(e) are the patterns from the cavity with a length of $865 \mathrm{~nm}$ corresponding to the resonant wavelengths 650 and $793 \mathrm{~nm}$, respectively. The brightness of the CL images corresponds to the emitted photon intensity. The line profiles of the two modes in Figs. 2(b) and 2(c) along the symmetrical axis of the resonator that parallels the $\mathbf{x}$ axis are shown in Figs. 2(f) and $2(\mathrm{~g})$, respectively. The patterns show cosinelike intensity variations inside the cavity. The following discussions show that the profiles near the edge cannot predict the real reflection phase shift due to the contribution of the backscattering electrons, with energy similar to the incident electrons. When the electron beam is incident on the
Ag surface inside the cavity, especially close to the reflectors, the backscattered electrons impinge on the Ag reflectors and excited SPPs contributing to the CL signal.

To determine the origin of the mode patterns, we calculated the modes for a cavity with $L=545 \mathrm{~nm}$ at $550 \mathrm{~nm}$ vacuum wavelength, corresponding to the case in Fig. 2(b), by using the finite-difference time-domain method. Figures 3(a) and 3(b) show the simulated patterns of the in-plane and out-of-plane components of the electric field, respectively. Here, we named the modes as $(m, n)$, where the integers $\mathbf{m}$ and $\mathbf{n}$ denote the number of intensity antinodes (or nodes) of the out-of-plane (or in-plane) component in the $\mathbf{x}$ and $\mathbf{y}$ directions, respectively. Therefore, Figs. 3(a) and 3(b) are assigned to the $(2,1)$ mode. Because of the $\mathbf{x}-\mathbf{y}$ symmetry of the CL microscopy, the patterns obtained in the experiment are the overlap of the $(m, n)$ and $(n, m)$ modes when $m \neq n$. The $\mathbf{x}-\mathbf{y}$ symmetry of the patterns can be broken by using light excitation. Figures 3(c) and 3(d) depict the numerical simulations of the overlap of the $(2,1)$ and $(1,2)$ modes of the in-plane and out-of-plane components, respectively. The difference of the modes of the in-plane and out-of-plane components originates from the different reflection phase shift. We calculated the reflection phase shift and reflectivity of SPPs by using a two-dimensional finite-difference time-domain method. Figure 3(e) shows the phase shifts and the intensity reflectivities of the in-plane and out-ofplane components of the electrical fields $\boldsymbol{E}_{x}$ and $\boldsymbol{E}_{z}$, respectively. Although the reflections are almost the same, the phase shifts are significantly different from one another and equal to $\sim 0$ and $\sim \pi$, respectively. A comparison of the
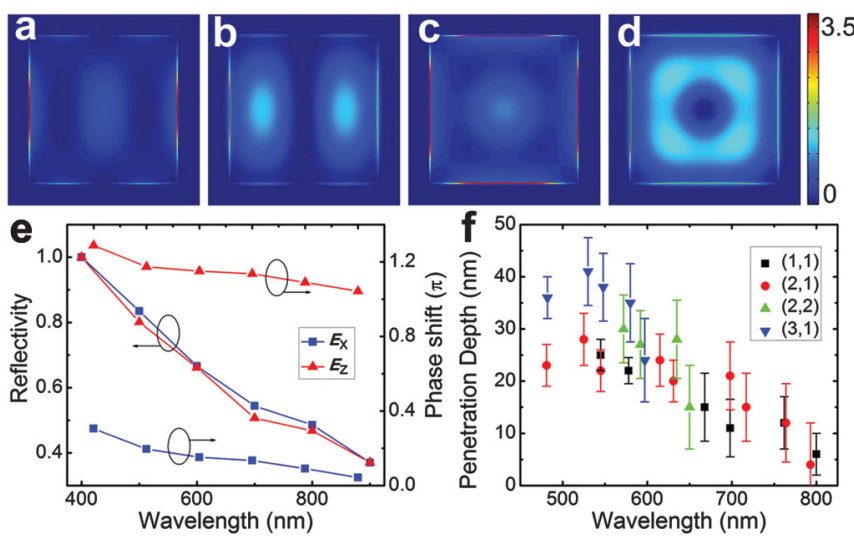

FIG. 3 (color). Calculated mode patterns of the out-of-plane (a),(c) and in-plane components (b),(d) of SPPs at $550 \mathrm{~nm}$ wavelength for the 545-nm-long cavity, respectively. (e) Reflectivities and reflection phase shifts of the in-plane component $\boldsymbol{E}_{x}$ and the out-of-plane component $\boldsymbol{E}_{z}$ of SPPs. (f) The penetration of SPPs into the cavity reflectors, calculated by comparing the measured resonant wavelength and physical cavity length based on Eq. (1). Error bars originate from the difference between the two orthogonal physical cavity side lengths. 
simulation and the experimental results shows excellent agreement between Figs. 2(b) and 3(d), indicating that the mode of the out-of-plane field component dominates the experimental results. This demonstrates the important characteristic of the localized excitation of SPPs by the electron beam, which locally excites the out-of-plane field component of SPPs. This conclusion is crucial to understanding the plasmonic mode in nanocavities excited by an electron beam.

For simplicity, we adopt an additional naming convention that $(m, n)$ represents also the $(n, m)$ mode or their overlap $m \neq n$. Thus, Figs. 2(b) and 2(c) are the composite patterns of the $(2,1)$ and $(1,1)$ modes, respectively. Figure 2(a) shows the corresponding modes of all resonant wavelengths of the 12 cavities. To understand the nature of these nanocavities, a simple analytical model is performed to investigate the experimental results. The multimode spectrum is accounted for by the normal modes of the square cavity, and the resonant condition of the nanocavity is given by

$$
L+2 \delta\left(\lambda_{\mathrm{SPP}}\right)=\sqrt{m^{2}+n^{2}} \frac{\lambda_{\mathrm{SPP}}}{2},
$$

where $\lambda_{\mathrm{SPP}}$ is the SPP wavelength and $\delta\left(\lambda_{\mathrm{SPP}}\right)$ is the penetration of SPPs into the Ag reflectors, which depend on the phase shift of plasmon reflection at the reflectors $(\Delta \phi)$, the size of the cavity, and the height of the reflectors. For the four lowest modes in Fig. 2(a), the values of $\delta\left(\lambda_{\mathrm{SPP}}\right)$ are shown in Fig. 3(f). The penetration depth is in the range of $4-41 \mathrm{~nm}$. The penetration increases with decreasing wavelengths in the measurement range, which is consistent with the calculation of phase shift in Fig. 3(e). The penetration also results in the structures at the edges, which are clearer for a shorter wavelength due to a larger $\delta$. The defocusing of the electron beam in the Ag reflectors blurs the structures. At the same time, the strong signal at the edges of the cavity can be attributed to the scattering of the SPPs on the surface of the reflectors excited by the scattering electrons in the Ag reflectors.

The plasmonic field distributions in the $\mathbf{x}$ and $\mathbf{y}$ directions are standing waves, and the distribution in the $\mathbf{z}$ direction has an exponential decay [2]. For the $(1,1)$ mode, according to Eq. (1) with the approximation of $\delta\left(\lambda_{\mathrm{SPP}}\right)=0$ and the definition of the mode volume [1], we can obtain the nanocavity's mode volume $V=$ $\lambda_{\mathrm{SPP}}^{3}\left(1+\left|\varepsilon_{m}^{\prime}\right|\right)\left(32 \pi \sqrt{\left|\varepsilon_{m}^{\prime}\right|}\right)^{-1}$, where $\varepsilon_{m}^{\prime}$ is the real part of the permittivity of Ag. For example, for a 320-nm-long cavity, the resonant wavelength in vacuum is $545 \mathrm{~nm}$ and $\varepsilon_{m}^{\prime}=-10.5$ [20]. Thus, we can obtain $V=0.035 \lambda_{\mathrm{SPP}}^{3}=$ $0.0049 \mu \mathrm{m}^{3}$.

We can obtain the $Q$ factors from Fig. 2(a) directly. The results are shown in Fig. 4 for the $(1,1),(2,1),(2,2)$, and $(3,1)$ modes. The highest $Q$ factor was obtained at $597 \mathrm{~nm}$ wavelength up to 22 for the mode $(3,1)$. The results shown in Fig. 4 represent the trade-off between the reflection and

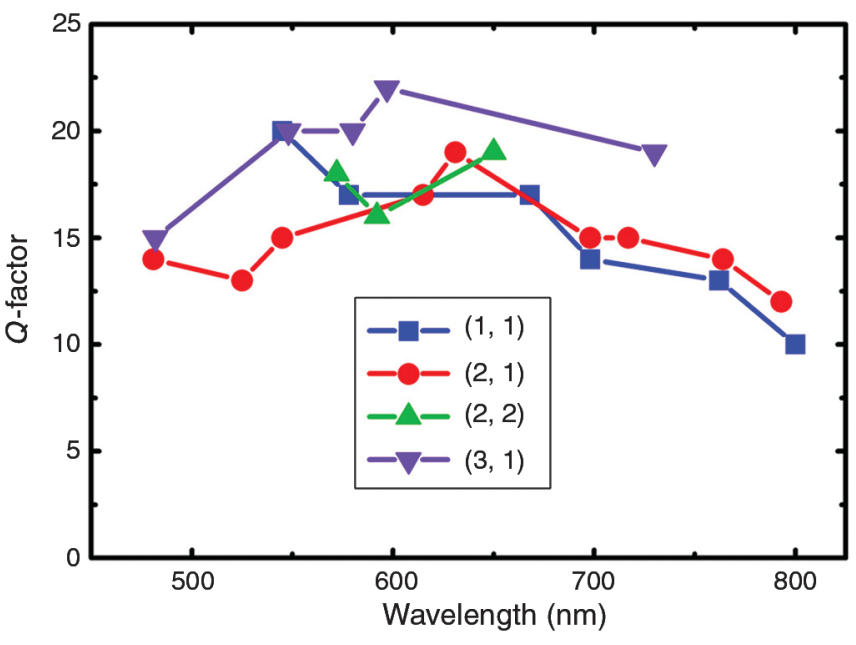

FIG. 4 (color). $Q$ factors directly obtained from the measured CL spectra corresponding to plasmonic cavity modes $(1,1)$, $(2,1),(2,2)$, and $(3,1)$, respectively.

propagation loss: The highest $Q$ factor appears at $\sim 600 \mathrm{~nm}$. Although the $Q$ factor is lower than that of dielectric cavities, the $Q / V$ ratio is comparable to general optical cavities due to the small modal volume. To obtain a higher $Q$ factor, we can increase the height of the reflector to improve reflectivity and lower the scattering coupling of the top corners of the reflectors [6].

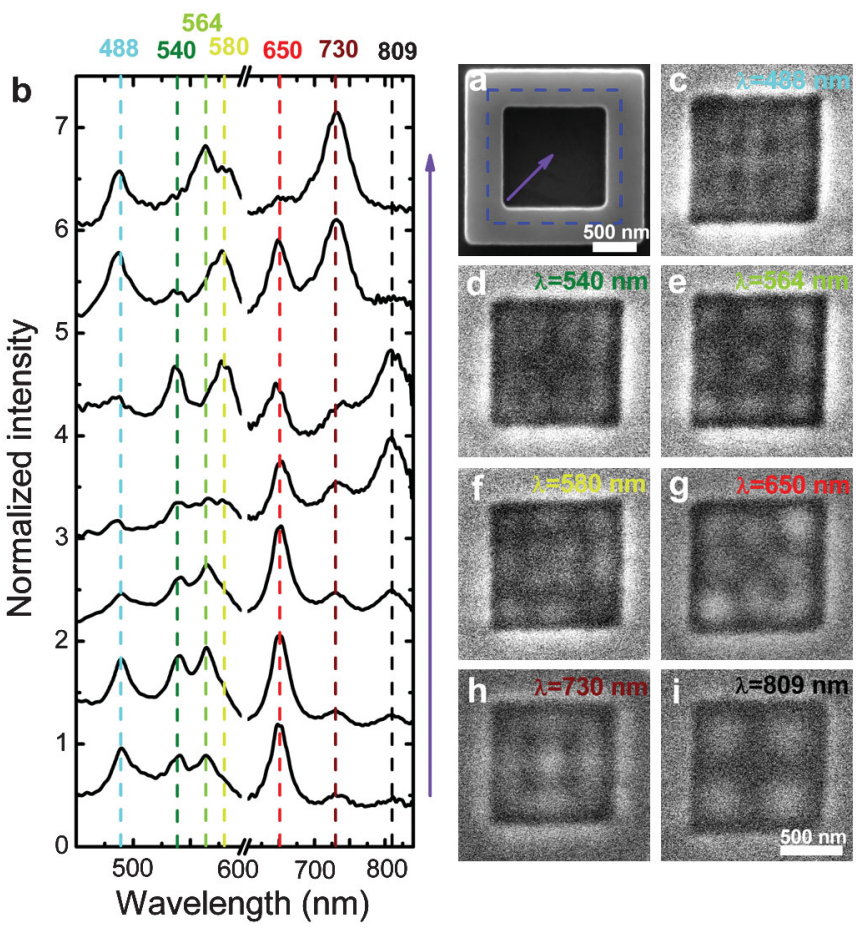

FIG. 5 (color). (a) Top-view SEM image of the cavity. (b) CL spectra for the electron beam positioned along the violet arrow. Spectra are offset vertically for clarity. The breaks at $600 \mathrm{~nm}$ mean two measurements. (c)-(i) Monochromatic CL images taken at the resonant wavelength. 
Compared with the plasmonic Fabry-Pérot nanocavity with the same cavity length, the boxing ring-shaped nanocavity usually has more resonant modes. Here, we used a 1140-nm-long nanocavity to obtain more resonant modes [Fig. 5(a)]. The electron beam is scanned with a step spacing of $110 \mathrm{~nm}$ from the vertex to the center of the square cavity along the violet arrow in Fig. 5(a), while the CL spectra at different points are shown in Fig. 5(b). Seven resonance wavelengths are observed at 488, 540, $564,580,650,730$, and $809 \mathrm{~nm}$ for the plasmonic modes $(4,3),(4,2),(3,3),(4,1),(3,2),(3,1)$, and $(2,2)$, respectively, according to Eq. (1) with the approximation of $\delta\left(\lambda_{\mathrm{SPP}}\right)=0$. The strong dependence of the spectra on the position reflects the multiple modal properties. The patterns of the modes at the resonant wavelengths are shown in Figs. 5(c)-5(i). Although increasing the cavity length results in a larger propagation loss, the patterns are still clear in the measurement range.

The differences between the nanocavity with metal reflectors and with single or periodic groove reflectors $[4,8]$ are manifested as follows. (i) The metal reflectors effectively confine the SPPs in the direction normal to the interface, which results in a redshifting of the resonant wavelength, especially for a small cavity. In our study, the resonant wavelength of the $(1,1)$ mode redshifts from 577 to $655 \mathrm{~nm}$ when the height of the reflectors is increased from 300 to $500 \mathrm{~nm}$ in the $350 \mathrm{~nm}$ side length cavity. The height of the reflectors therefore provides another degree of freedom to control the resonant wavelength. (ii) The phase shifts of the out-of-plane and in-plane field components on reflection are considerably different. For the normal incident, the difference of the phase shifts between the metal reflector and the groove reflector is $\sim \pi$; that is, the phase shift of the out-of-plane or in-plane component is $\sim 0 / \pi$ for the groove reflector. The difference in phase shift results in differences of the mode patterns and boundary conditions. (iii) The reflectivity of the metal reflectors is not sensitive to the incident angle, which is another reason to obtain more resonant modes.

In conclusion, we analyzed the plasmonic modes of the boxing ring-shaped nanocavities. A comparison between the experimental results and the theoretical simula tions proved that the electron beam locally excites the out-of-plane field component of SPPs, while the observed plasmonic modes correspond to the modes of the out-ofplane field component. Because of the use of metal reflectors, the total size of the cavity is small with many plasmonic resonant modes. Numerous applications, such as plasmonic filter, nanolaser, and efficient light-emitting devices, can be expected to arise from these developments.

This work is supported by NSFC $(10804003,61036005$, and 11074015), the national 973 program of China (2007CB936203 and 2009CB623703), MOST, and NSFC/RGC (N HKUST615/06).

\section{*jszhang@pku.edu.cn} †yudp@pku.edu.cn

[1] J. S. Foresi et al., Nature (London) 390, 143 (1997).

[2] H. Raether, Surface Plasmons on Smooth and Rough Surfaces and on Gratings (Springer, Berlin, 1988).

[3] W.L. Barnes, A. Dereux, and T.W. Ebbesen, Nature (London) 424, 824 (2003).

[4] C. E. Hofmann et al., Nano Lett. 7, 3612 (2007).

[5] M. Kuttge, E. J. R. Vesseur, and A. Polman, Appl. Phys. Lett. 94, 183104 (2009).

[6] V. J. Sorger et al., Nano Lett. 9, 3489 (2009).

[7] E. J. R. Vesseur, F. J. García de Abajo, and A. Polman, Nano Lett. 9, 3147 (2009).

[8] M. Kuttge, Ph.D. thesis, FOM Institute for Atomic and Molecular Physics (AMOLF), 2009.

[9] H. Ditlbacher et al., Phys. Rev. Lett. 95, 257403 (2005).

[10] B. J. Wiley et al., Nano Lett. 8, 3023 (2008).

[11] B. Min et al., Nature (London) 457, 455 (2009).

[12] S. I. Bozhevolnyi et al., Nature (London) 440, 508 (2006).

[13] J. T. van Wijngaarden et al., Appl. Phys. Lett. 88, 221111 (2006).

[14] M. V. Bashevoy et al., Nano Lett. 6, 1113 (2006).

[15] E. J. R. Vesseur et al., Nano Lett. 7, 2843 (2007).

[16] P. Wagner et al., Langmuir 11, 3867 (1995).

[17] L. Chai and J. Klein, Langmuir 23, 7777 (2007).

[18] G. Leveque, C. G. Olson, and D. W. Lynch, Phys. Rev. B 27, 4654 (1983).

[19] G. Fuster et al., Phys. Rev. B 42, 7322 (1990).

[20] E. D. Palik, Handbook of Optical Constants of Solids (Academic Press, New York, 1985). 